\title{
Symmetric Deflection of CFRP-Based Polymeric Laminate
}

\author{
Wenyi Lin ${ }^{a}$, Minoru Sasaki ${ }^{b}$, Hirohisa Tamagawa ${ }^{c}$ \\ Department of Human and Information Systems, Faculty of Engineering, Gifu University, 1-1 \\ Yanagido, Gifu, Gifu, 501-1193 Japan \\ ap3128035@edu.gifu-u.ac.jp bsasaki@gifu-u.ac.jp(corresponding author) ctmgwhrhs@gifu-u.ac.jp
}

Keywords: Carbon fibres, Laminate, Electrical properties, Thermal properties, Deformation

\begin{abstract}
Unlike well-investigated polymer-based soft actuators such as gel, Ionic Polymer-Metal Composite, conducting polymer, a CFRP-based polymeric laminate possesses quite distinguished properties. It was previously reported that a CFRP-PVC laminate exhibited two-way deflection in accordance with environmental temperature, but it was asymmetric deflection due to the asymmetric laminate structure. In this study, we successfully fabricated a new CFRP-based polymeric laminate which can exhibit symmetric deflection. Despite such a successful outcome, there was large room to improve the degree of its deflection. Improvement of temperature control method for the newly fabricated CFRP-based polymeric laminate resulted in enhancement of the degree of its deflection.
\end{abstract}

\section{Introduction}

The study of soft actuators has attracted considerable attention of researchers and engineers. There is widespread ongoing research on electroactive polymer-based soft actuators, especially hydrogels $[1,2]$, ionic polymer-metal composites (IPMC) $[3,4]$, and conducting polymers [5,6] are being intensively investigated. These polymers have unique characteristics that are useful for actuator use. However, they also have undesirable charcteristics. For example, hydrogel shape change speed is considerably slow, IPMC hardly generates high force, and conducting polymers are typically electrically activated in a highly wet state. A practical soft actuator should exhibit rapid shape change and generate high force without the use of a liquid, which is not preferred because of the difficulty in handling a wet actuator.

A CFRP-PVC laminate is a polymeric laminate that is fabricated by gluing a carbon fiber reinforced plastic (CFRP) plate to a polyvinylchloride (PVC) plate [7]. CFRP causes Joule heating by the application of voltage. Because of the disparity in the coefficients of thermal expansion between CFRP and PVC, the CFRP-PVC laminate exhibits deflection by imposing a voltage on the CFRP portion. The shape change is considerably rapid, and the CFRP-PVC laminate can generate an adequately high force. Moreover, the CFRP-PVC laminate is a solid-state material. Although the deflection of the CFRP-PVC laminate is thermally induced. Therefore, the CFRP-PVC laminate is regarded as an electroactive polymer. Although the CFRP-PVC laminate possesses useful characteristics as a soft actuator, it has some limitations. For instance, the CFRP-PVC laminate assumes a deflected shape in the PVC plate direction at room temperature and the Joule heat generated by the voltage application straightens the CFRP-PVC laminate. However, because of the asymmetric structure of CFRP-PVC laminate, the CFRP-PVC laminate hardly deflects in the CFRP plate direction. That is, the CFRP-PVC laminate exhibits asymmetric rather than symmetric deflection.

In this study, we attempted to fabricate a polymeric laminate-type actuator that can exhibit symmetric deflection, and quantitatively evaluated its actuator performance.

\section{Previous work and current research aim}

In previous work, we previously fabricated a polymeric laminate-type actuator that was equipped with a cooling system [8]. The polymeric laminate consisted primarily of a CFRP plate and an acryl plate. We begin by explaining the fabrication process.

An acryl plate was machined to possess three grooves on its surface as shown in Fig.1. 


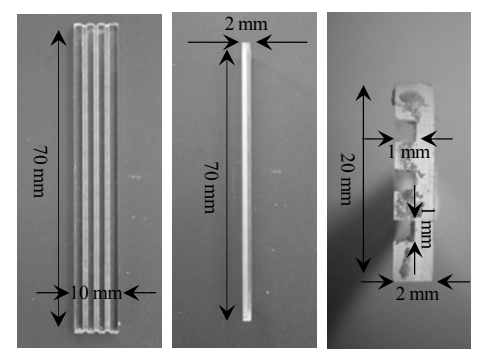

Fig.1 A grooved acryl plate

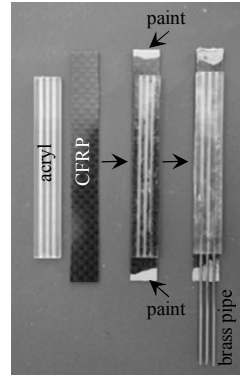

Fig. 2 Fabrication procedure of CFRP-Acryl laminate

As illustrated in Fig. 2, the grooved acryl plate was glued to a CFRP plate; the laminate is hereafter called CFRP-Acryl laminate. Both edges of the CFRP-Acryl laminate's CFRP plate were coated with Dotite, an electrically conductive paint (Fujikura Kasei Co., Ltd., Japan). The paint served as a flexible electrode, and three brass pipes were inserted into the grooves (see Fig. 2).

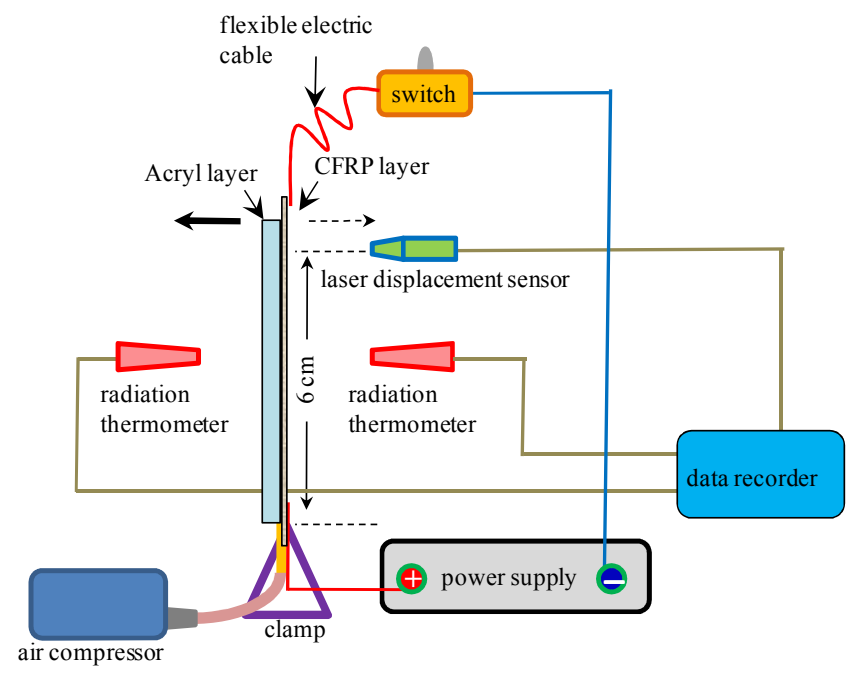

Fig. 3 Experimental setup for measuring tip displacement of the CFRP-Acryl laminate

Fig. 3 shows an illustration of the experimental setup for measuring the tip displacement of the CFRP-Acryl laminate. The CFRP-Acryl plate was vertically clamped. The painted edges of the CFRP plate were connected to the terminals of a power supply. An air compressor was connected to the brass pipes of the CFRP-Acryl laminate for introducing air through the grooves. Immediately after a constant voltage was imposed on the CFRP plate, Joule heat was generated. The Joule heat caused a larger expansion of the acryl plate than the CFRP plate, resulting in the deflection of the CFRP-Acryl laminate in the direction indicated by the dashed arrow in Fig. 3. Turning off the voltage restored the CFRP-Acryl laminate to its initial straight shape because of natural convection cooling. Photos of this process are shown in Fig. 4. We observed that pumping air through the grooves of the CFRP-Acryl laminate by using the air compressor accelerated cooling, which resulted in faster shape restoration. This CFRP-Acryl laminate exhibited a two-way shape change, and the shape-change speed could be controlled by imposing a higher voltage or forcing air through the grooves. However, the CFRP-Acryl laminate did not exhibit leftward deflection as indicated by the thick arrow in Fig. 4.

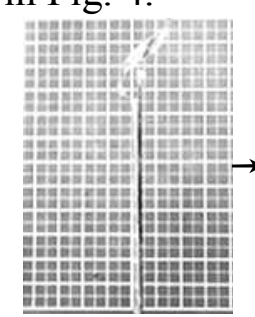

under $0 \mathrm{~V}$

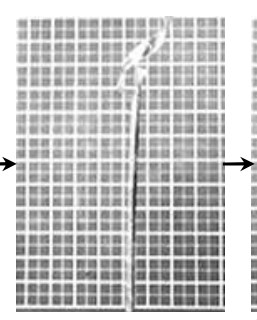

under $4 \mathrm{~V}$

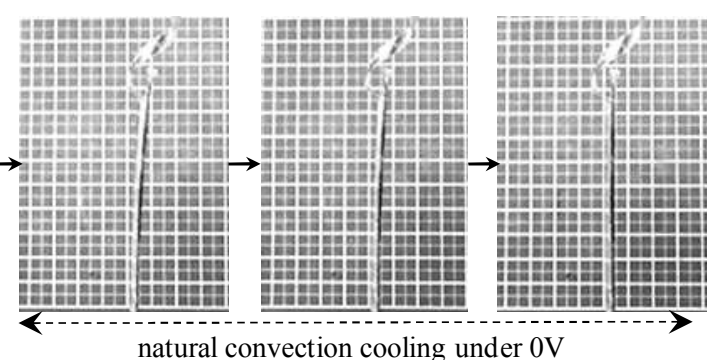

natural convection cooling under $0 \mathrm{~V}$

Fig. 4 Bending behavior of the CFRP-Acryl laminate 
Therefore, the shape change of the CFRP-Acryl laminate was asymmetric. Symmetric deflection of the CFRP-Acryl laminate could broaden its potential usefulness; thus, in this study we fabricate a new CFRP-based polymeric laminate-type actuator that exhibits symmetric deflection.

\section{Specimen preparation}

First, a grooved acryl plate was prepared with the same structure as the plate shown in Fig. 1. The surface opposite to the grooved surface was crazed and subsequently sandblasted [9-12] to yield a highly surface-roughened acryl plate which was coated with Dotite. It should be noted that the combined treatment of crazing and sandblasting the surface resulted in the formation of a Dotite coat that strongly adhered to the acryl plate [13]. Another piece of the same acryl plate was prepared. The two pieces of the Dotite-coated acryl plates were glued to a CFRP plate as shown in Fig. 5. Six brass pipes were inserted into each groove of the acryl plates, and gas hoses were connected to the brass pipes as shown in Fig. 6. The specimen shown in Fig. 6 is hereafter called DACAD1. For convenience of discussion, the two acryl plates of DACAD1 are designated as Acryl-A and Acryl-B, respectively.

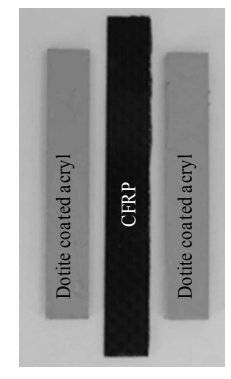

(a)

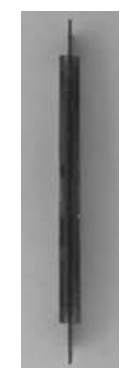

(b)

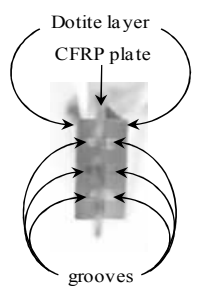

(c)

Fig. 5 (a) Two pieces of the Dotite coated grooved acryl plates and a CFRP plate (b) An assembly fabricated by gluing the three plates together (c) Cross section of the assembly
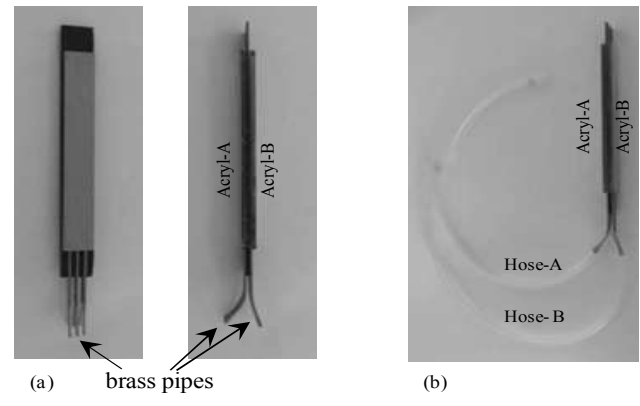

Fig. 6 DACAD1

(a) Six brass pipes were inserted into each groove of the assembly. (b) Gas hoses were hooked up to the brass pipes.

\section{Measurements}

The tip deflection of DACAD1 was measured using the setup shown in Fig. 3. DACAD1 was vertically clamped. Hose-A and Hose-B were attached to separate air compressors in order to operate them independently. A constant voltage of $4.5 \mathrm{~V}$ was imposed on the Dotite layer of DACAD1. The tip displacement of DACAD1 was measured with a laser displacement sensor as a function of time, and the tip displacement was later converted into curvature to provide a more general sense of the deflection. The surface temperatures of Acryl-A and Acryl-B were measured with radiation thermometers along with the tip displacement.

\section{Results and discussion}

\subsection{Deflection of DACAD1}

Fig. 7 shows the time dependence of the DACAD1 curvature. The Dotite layer of Acryl-A was charged with $4.5 \mathrm{~V}$ from time $\mathrm{t}=0 \mathrm{~s}$ to $t_{a}$, where the Dotite layer served as a flexible electrode and a resistor for generating Joule heat that resulted in the deflection of DACAD1 in the direction of Acryl-B. The voltage was turned off at $\mathrm{t}=t_{a}$, and natural convection cooling of DACAD1 was allowed from $t_{a}$ to $t_{b}$. The same voltage of $4.5 \mathrm{~V}$ was imposed on the Dotite layer of Acryl-B from $t_{b}$ to $t_{c}$, resulting in the deflection of DACAD1 in the direction of Acryl-A. The voltage was turned off at $\mathrm{t}=t_{c}$, and natural convection cooling was again allowed for the remaining time. A symmetric deflection curvature with respect to $0.0000 \mathrm{~mm}^{-1}$ can be clearly observed in Fig. 7. The DACAD1 deflection was primarily caused by a disparity in the thermal expansion degree between Acryl-A and Acryl-B. Such a disparity is caused by the temperature difference between Acryl-A and AcrylB, i.e., Surface temp. (Acryl-A) - Surface temp. (Acryl-B), which is shown in Fig. 8. The curvature behavior agrees well with the behavior of temperature difference. 

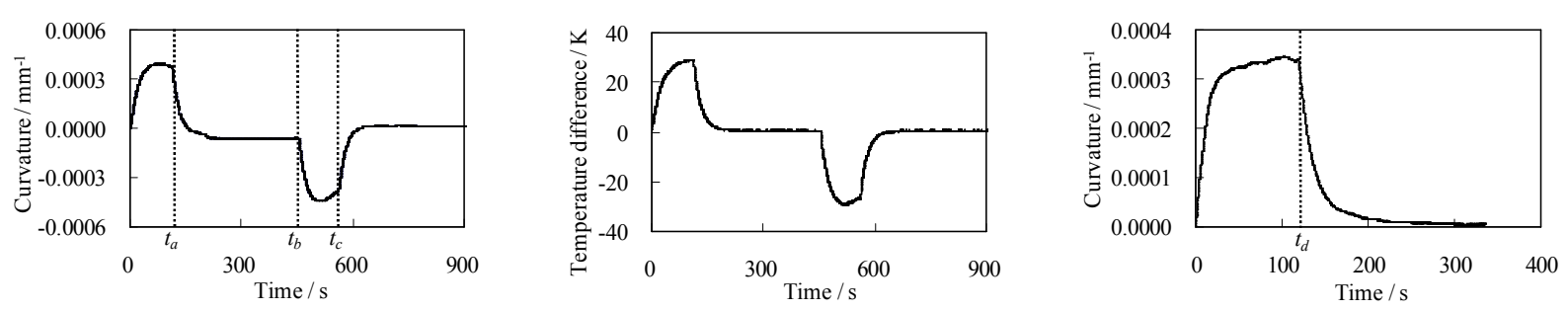

Fig. 7 Curvature vs. Time Fig. 8 Surface temperature vs. Time Fig.9 Curvature in cooling process

The natural convection cooling only considerably slowly restored the DACAD1 to its original shape, as can be clearly observed in Fig. 7. Therefore, cooling by using the air compressor was performed after inducing the deflection of DACAD1, because it was previously reported that the shape restoration of the CFRP-Acryl laminate was successfully accelerated by air compressor cooling [8]. Fig.9 shows the time dependence of the DACAD1 curvature. Again, a voltage of $4.5 \mathrm{~V}$ was imposed on the Dotite layer of Acryl-A from $\mathrm{t}=0 \mathrm{~s}$ to $t_{d}$, and then the air compressor connected to Acryl-A was turned on. The air passed through the grooves between the Acryl-A and CFRP plates. Acryl-A was expected to effectively cool down and result in rapid shape restoration. Contrary to our expectations, the shape restoration speed was not rapid. Fig.10 (a) shows the surface temperature of the Acryl-A and the Acryl-B for initial $400 \mathrm{~s}$, when the measurement in Fig. 8 was carried out. Fig.10 (b) shows the surface temperature of the Acryl-A and the Acryl-B, too, when the measurement in Fig.9 was carried out. It is evident that the surface temperature of Acryl-A and Acryl-B under air compressor cooling decreased faster than that observed by natural convection cooling. However, the air compressor cooling did not considerably affect the shape restoration speed (compare Fig.9 with Fig. 7). The deflection of DACAD1 was induced by the disparity in the thermal expansion degree between Acryl-A and Acryl-B. As can be seen in Fig.10 (a) and (b), the cooling process started at approximately the same time, $\mathrm{t}=t_{a} \sim t_{d}$, for both cases. The disparity in the temperature between Acryl-A and Acryl-B became negligible at the time indicated by the arrows in Fig.10 (a) and (b), and the shape of DACAD1 was fully restored at those moments. The duration of cooling required for the shape restoration of DACAD1 by natural convection cooling and by air compressor cooling was given by $\Delta t$ and $\Delta t^{\prime}$, respectively, where $\Delta t$ and $\Delta t$ ' are exactly defined in Fig.10. $\Delta t$ ' is only slightly shorter than $\Delta t$, although entire body of the DACAD1 was cooled faster by the air compressor cooling than by the natural convection cooling. It is because the air compressor cooling affect not only Acryl-A but also Acryl-B simultaneously. Therefore, the temperature disparity between Acryl-A and Acryl-B did not become negligible so fast even by the air compressor cooling, resulting in only slight improvement of shape restoration speed of DACAD1. In other words, as long as the temperature disparity between Acryl-A and Acryl-B is negligible, DACAD1 assumes a straight shape, even if the entire body of DACAD1 is maintained at a high temperature. This fact also suggests that prolonged voltage application on Acryl-A would not necessarily induce a larger deflection of DACAD1 because Acryl-B could be also be considerably heated along with Acryl-A owing to heat conduction from the Dotite layer on Acryl-A.

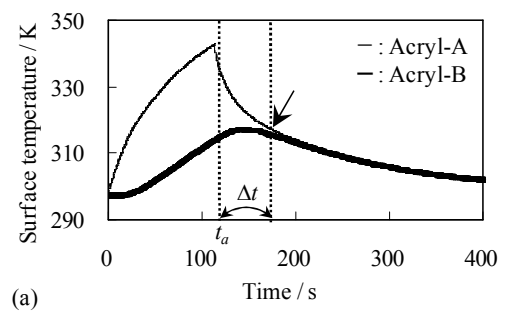

(a) By natural convection cooling

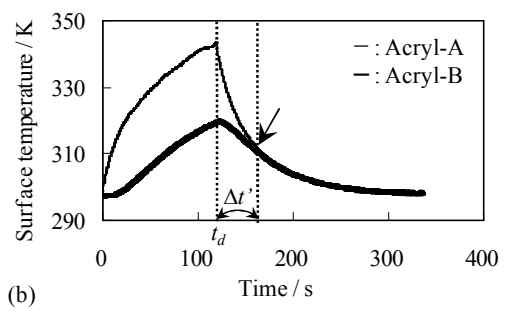

(b) By the air compressor cooling

Fig.10 Time dependence of surface temperature of Acryl-A and Acryl-B

Therefore, it is concluded that the large temperature difference between Acryl-A and Acryl-B should be forcibly realized for achieving larger deflection of DACAD1. For realizing a large temperature difference between Acryl-A and Acryl-B, a technique for independently controlling 
their temperatures is required; the temperature of one acryl should be forcibly increased, while the other acryl should be forcibly cooled. Therefore, we fabricated a new CFRP-based laminate that sufficed the requirements for inducing a large deflection.

\subsection{Improvement of DACAD1 deflection}

A new CFRP-based laminate was fabricated. A grooved acryl plate designated as Acryl- $\alpha$ was glued to a CFRP plate as shown in Fig.11 (a). Three fine brass pipes were inserted into the grooves; one of which was U-shaped and connected the right and left channels. Two thick brass pipes were placed over the fine brass pipes at the bottom of the laminate. These brass pipes connected the right groove to the left groove, forming a single channel as indicated by the dashed arrows in Fig.11 (a). The central groove was not used in this experiment. The two brass pipes at the bottom were respectively designated as Inlet- $\alpha$ and Outlet- $\alpha$. The surface of Acryl- $\alpha$ was coated with Dotite (see Fig.11 (b)). The same structure was created at the opposite side of the CFRP plate, and the acryl plate glued to this side was designated as Acryl- $\beta$. The two thick brass pipes at the bottom were respectively designated as Inlet- $\beta$ and Outlet- $\beta$ (see Fig.11 (b)). The resultant laminate is hereafter called DACAD2.

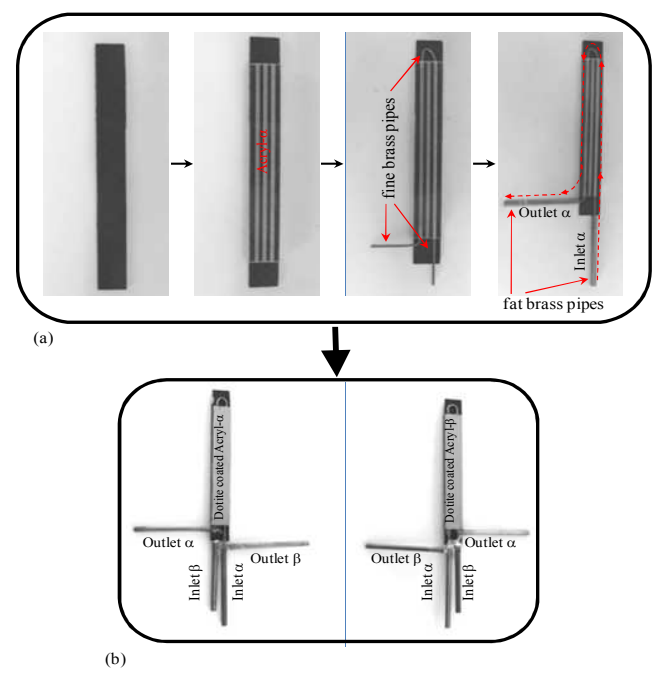

Fig.11

DACAD2

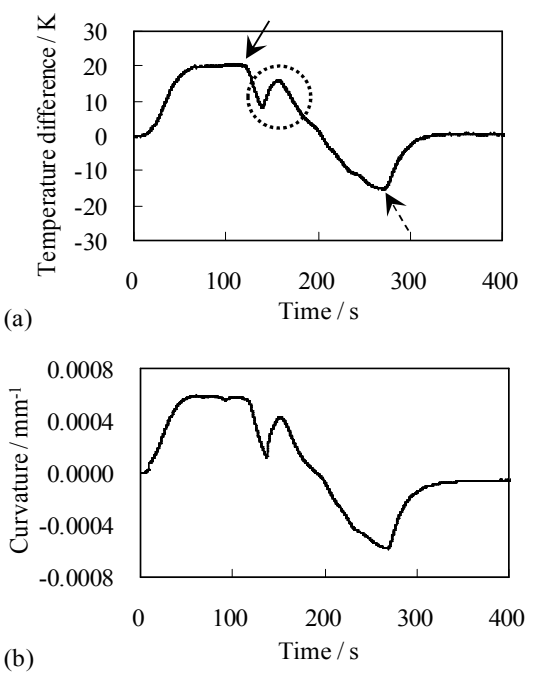

Fig.12 Time dependence of the DACAD2 curvature

The temperature of Acryl- $\alpha$ and Acryl- $\beta$ were independently controlled by supplying either hot water $\left(75^{\circ} \mathrm{C}\right)$ or cold water $\left(15^{\circ} \mathrm{C}\right)$ from Inlet- $\alpha$ and Inlet- $\beta$. Although DACAD2 required the use of a liquid (water), the water was completely confined in DACAD2; therefore, DACAD2 was not in the wet state. Hence, the use of water is not an obstacle in the use of DACAD2, unlike the previously mentioned conducting polymer-based actuator. The deflection of DACAD2 was measured using the setup shown in Fig. 3. Fig.12 (a) shows the time dependence of the surface temperature difference between Acryl- $\alpha$ and Acryl- $\beta$; i.e., Surface temp. (Acryl- $\alpha$ ) - Surface temp. (Acryl- $\beta$ ). Initially, hot water was circulated from Inlet- $\alpha$ through Outlet- $\alpha$, while cold water was circulated from Inlet- $\beta$ through Outlet- $\beta$. Hence, the surface temperature difference increased. The curvature of DACAD2 is shown in Fig.12 (b), which increased and reached a high of $0.00055 \mathrm{~mm}^{-1}$, higher than the peak curvature of DACAD1 shown in Fig.7. Thus, an effective temperature difference was realized in DACAD2. Cold water was circulated from Inlet- $\alpha$ through Outlet- $\alpha$ at the time indicated by the solid arrow in Fig.12 (a), while hot water was circulated from Inlet- $\beta$ through Outlet- $\beta$. Then, the curvature of DACAD2 started decreasing. We intentionally induced a temperature disturbance in DACAD2 by momentarily adjusting the flow rate of hot water as indicated by the dotted circle in Fig.12 (a). The curvature of DACAD2 was disturbed by the temperature change, and it passed through zero curvature and continued decreasing. Its lowest peak was at $-0.00053 \mathrm{~mm}^{-1}$, and its magnitude was almost same as that of the highest peak. Therefore, it is concluded that symmetric deflection can be obtained. Natural convection cooling was allowed 
from the time indicated by the dashed arrow in Fig.12 (a). The deflection of DACAD2 perfectly followed the temperature behavior. Such a strong obedience of the DACAD2 curvature to the temperature difference was observed even when the temperature disturbance was applied.

\section{Conclusion}

A CFRP-based laminate-type actuator that exhibited symmetric deflection was fabricated by gluing two acryl plates to both sides of a CFRP plate. It was experimentally confirmed that symmetric deflection of the actuator could be achieved by controlling the temperature of individual layers of the laminate. Circulation of both hot and cold water through the CFRP-based laminatetype actuator enabled the appropriate control of the laminate temperature, resulting in its symmetric deflection. Although a liquid (water) was employed to control the laminate temperature, the liquid was completely confined in the laminate. Therefore, water was not an obstacle in the use of the CFRP-based laminate, unlike the wet-type actuator.

\section{Acknowledgement}

This work was financially supported by Ministry of Education, Culture, Sports, Science and Technology for the financial support under the Knowledge Cluster Initiative the Second Stage Tokai Region Nanotechnology Manufacturing Cluster and the Air Force Research Laboratory under the agreement number FA23861014119.

\section{References}

[1] T. Tanaka, I. Nishio, S.T. Sun and S. Ueno-Nishio. Collapse of Gels under an Electric Field. Science, 218, 467-469, 1982.

[2] Y. Osada, H. Okuzaki and H. Hori. A Polymer Gel with Electrically Driven Motility. Nature, 355, 242-244, 1992.

[3] K. Oguro, Y. Kawami and H. Takenaka. Bending of an ion-conducting polymer filmelectrode composite by an electric stimulus at low voltage. Trans. J. Micromach. Soc., 5, 2730, 1992.

[4] K. Oguro, K. Asaka and H. Takenaka. Polymer Film Actuator Driven by Low Voltage. in Proceedings of 4th International Symposium on Micro Machine and Human Science, 39-40, Nagoya, 1993.

[5] K. Sugiyama, K. Ishii and K. Kaneto. A Control Method of a Biomimetic Actuator Using Electroconductive Polymer. in Proceeding of SCIS \& ISIS, 1687-1692, Nagoya, 2008.

[6] A.S. Lee, S.F. Peteu1, J.V. Ly, A.A.G. Requicha, M.E. Thompson and C. Zhou. Actuation of polypyrrole nanowires. Nanotechnol., 19, 165501, 2008.

[7] H. Tamagawa, K. Kikuchi and G. Nagai. Mechanical characteristics of a thermo-responsive two-way shape change polymeric laminate. Sens. Actuators A: Physical, 163, 356-362, 2010.

[8] H. Tamagawa, W. Lin, S. Inaba and M. Sasaki Electroactive thermo-responsive polymeric actuator equipped with a built-in air cooling system. in Proceedings of Sead 23 (to be submitted), Aichi, Japan, 2011.

[9] A. Takeno, N. Nakagaki and M. Miwa. Anisotoropic transparency of polystyrene film with carazes. Adv. Composite Mater., 7, 35-46, 1998.

[10] F. Noguchi, Y. Iwashige, A. Takeno and M. Miwa. View field selective film of Monocomponent polyester composites. J. Advanced Science, 13, 382-385, 2001.

[11] A. Takeno, M. Yoshimura, M. Miwa and T. Yokoi. Anisotorpic Electric Conductivity and Light Transmittance of Polypyrrole/Poly(vinylidene fluoride) Composited Film by Polymerization in Craze. SEN'I GAKKAISHI (in Japanese), 57, 301-305, 2001.

[12] A. Takeno, T. Ozeki, M. Miwa and T. Yokoi. Periodic Micro-necking of Polyster Filament. SEN'I GAKKAISHI (in Japanese), 63, 172-176, 2007.

[13] H. Tamagawa, W. Lin, K. Kikuchi and M. Sasaki. Bending control of Nafion-based electroactive polymer actuator coated with multi-walled carbon nanotubes, Sens. \&Actuators B:chem. 156, 375-382, 2011. 